\title{
Dolor del Músculo Piramidal en pacientes oncológicos. Tratamiento dirigido por Ultrasonido sin Hidrodisección Perineural del Ciático. Presentación de 10 casos.
}

\section{Pyramidal Muscle Pain in cancer patients. Treatment directed by Ultrasound without Perineural Hydrodissection of the Sciatic. Presentation of 10 cases.}

*Correspondencia:

mcvallejom@hotmail.es

Teléfono [593] 043718700

Conflicto de intereses: Los autores declaran no tener conflictos de intereses.

Fondos: Ver la página 147

Recibido: 5 Febrero 2017

Aceptado: 5 Febrero 2018

Publicado: 30 Agosto 2018

Membrete bibliográfico:

Vallejo $M$, Lino $N$, Palma $M$ Guamán J. Dolor del músculo piramidal en pacientes oncológicos. Tratamiento dirigido por ultrasonido sin hidrodisección perineural del ciático. Presentación de 10 casos. Rev. Oncol. Ecu 2018;28(2):138-149.

DOI: https://doi.org/10.33821/201

Copyright Vallejo, et al. Este artículo es distribuido bajo los términos de Creative Commons Attribution License, el cual permite el uso y redistribución citando la fuente y al autor original.

\section{Mariana Vallejo Martínez* iD, Nancy Lino1, M. Palma², J. Guamán1.}

1. Servicio de Dolor y cuidados Paliativos del Instituto Oncológico Nacional "Dr. Juan Tanca Marengo" Solca, Guayaquil-Ecuador.

2. Postgrado de Anestesia de la Universidad de Especialidades Espíritu Santo, Guayaquil-Ecuador.

\section{Resumen}

Introducción: El síndrome del músculo piramidal constituye el conjunto de signos y síntomas originados por la compresión del nervio ciático en su salida de la pelvis a través del canal situado entre el músculo piramidal y el gémino superior. Se presenta una serie de casos de pacientes oncológicos con este síndrome con el objetivo de describir el resultado terapéutico de la infiltración con esteroides en el músculo piramidal sin hidrodisección perineural del ciático.

Métodos: Se realizó un estudio prospectivo de 10 casos, evaluados entre Febrero de 2014 y Mayo del 2015, en el servicio de dolor y cuidados paliativos del Instituto Oncológico Nacional "Dr. Juan Tanca Marengo"-Solca-Guayaquil. Ingresaron al estudio pacientes con cáncer de cualquier etiología y criterios diagnósticos de síndrome piramidal. Los pacientes fueron tratados con infiltraciones de corticoides sin hidrodisección perineural del ciático, dirigidas por ultrasonido del músculo piramidal afecto y terapia física.

Resultados: Se incluyen 10 casos de pacientes con síndrome piramidal (9 mujeres). Con rangos de edad que entre 50 y 70 años. El lado afectado con mayor frecuencia fue el derecho en 5 casos. En 8 casos la Resonancia magnética fue normal y en 2 casos se describió hipertrofia muscular. El procedimiento de infiltración se realizó sin complicaciones y los pacientes recibieron adicionalmente fisioterapia. El EVA inicial fue $>6$ en 8 pacientes y 2 casos con EVA de 5. Los pacientes tuvieron una limitación funcional de un 10-20\%. El EVA postbloqueo disminuyó hasta 0-1 en todos los casos. 
Conclusión: En la presente serie de casos el síndrome piramidal fue controlado satisfactoriamente con infiltración de esteroides sin hidrodisección perineural del ciático y terapia física.

Palabras Claves: SÍNDROME DEL MÚSCULO PIRIFORME, CIÁTICA, INFILTRACIÓN, ULTRASONOGRAFÍA INTERVENCIONAL.

DOI: $10.33821 / 201$

\section{Abstract}

Introduction: Pyramidal muscle syndrome is a signs and symptoms set caused by sciatic nerve compression and its exit from the pelvis through the channel between the pyramidal muscle and the superior genus. We present a series of cases of oncological patients with this syndrome with the aim of describing the therapeutic result of steroid infiltration in the pyramidal muscle without perineural hydrodissection of the sciatic nerve.

Methods: A prospective study of 10 cases, evaluated between February 2014 and May 2015, in the pain and palliative care service of the National Oncological Institute "Dr. Juan Tanca Marengo "-SolcaGuayaquil. Patients with cancer of any etiology and diagnostic criteria of pyramidal syndrome entered the study. The patients were treated with corticosteroid infiltrations without perineural hydrodissection of the sciatic nerve, directed by pyramid-shaped muscle ultrasound and physical therapy.

Results: Ten cases of patients with pyramidal syndrome ( 9 women) are included. With age ranges between 50 and 70 years. The most affected side was the right in 5 cases. In 8 cases the magnetic resonance was normal and in 2 cases muscle hypertrophy was described. The infiltration procedure was completed without complications and the patients received additional physiotherapy. The initial EVA was $>6$ in 8 patients and 2 cases with VAS of 5 . The patients had a functional limitation of 10$20 \%$. The post-blocking EVA decreased to $0-1$ in all cases.

Conclusion: In the present series of cases the pyramidal syndrome was successfully controlled with steroid infiltration without perineural hydrodissection of the sciatic and physical therapy.

Keywords: PIRIFORM MUSCLE SYNDROME, SCIATICA, INFILTRATION, INTERVENTIONAL ULTRASONOGRAPHY.

DOI: $10.33821 / 201$

\section{Introducción}

El síndrome del músculo piramidal, es el conjunto de signos y síntomas originados por la compresión del nervio ciático en su salida de la pelvis a través del canal situado entre el músculo piramidal y el gémino superior [1] (Figura 1). Este síndrome aparece en pacientes activos durante la cuarta o quinta década de vida, más frecuente en mujeres, la relación $3 / 1$ [2-3]. Este síndrome es responsable de más del $65 \%$ de casos de lumbociática crónica no discal. 
Figura 1. Síndrome piriforme.

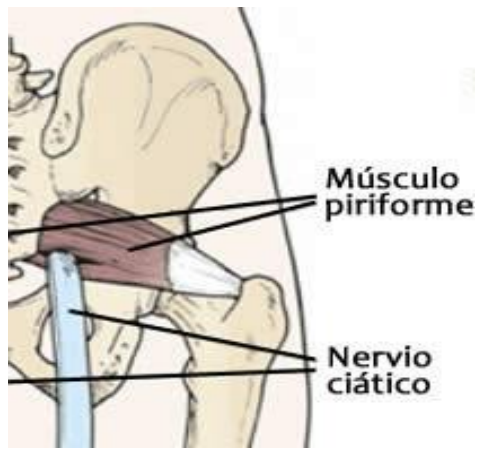

El nervio ciático pasa por o cerca del músculo piriforme o también llamado piramidal. El diagnóstico del síndrome Piramidal tiene 6 componentes: 1) Antecedentes de traumatismo en región glútea o sacroilíaca; 2) Presencia de dolor en la región sacroilíaca, en la escotadura ciática o en el trayecto del músculo piriforme que puede irradiarse por la extremidad inferior y dificultad para la marcha; 3) Exacerbación de la sintomatología dolorosa con la flexión de raquis o la carga de peso; 4) Palpación de una masa dolorosa sobre el piriforme; 5) Signo de Lasegue positivo y; 6) Atrofia de glúteos [4].

Existen 3 maniobras para evidenciar los signos clínicos útiles en el diagnóstico de este síndrome, los cuales son [5]: 1) Maniobra de Freiberg: Rotación forzada del músculo extendido que pone en tensión el músculo piriforme y se desencadena dolor en la zona glútea (Figura 2); 2) Maniobra de PACE: La abducción contra resistencia de la cadera afectada, con el paciente sentado o en decúbito lateral, genera el dolor (Figura 3); 3) Maniobra de Beatty: con el paciente en decúbito lateral sobre el lado sano y con la cadera flexionada realiza una abducción del muslo afectado, manteniendo esa posición unos segundos, lo que desencadena el dolor en la zona glútea por contracción selectiva del piriforme (Figura 4).

Figura 2. Maniobra de Freiberg

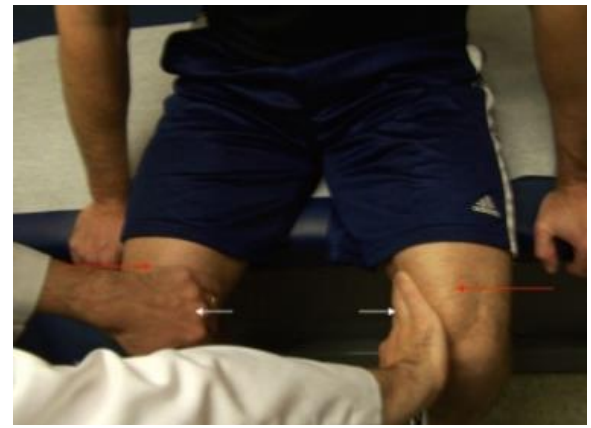


Figura 3. Maniobra de PACE

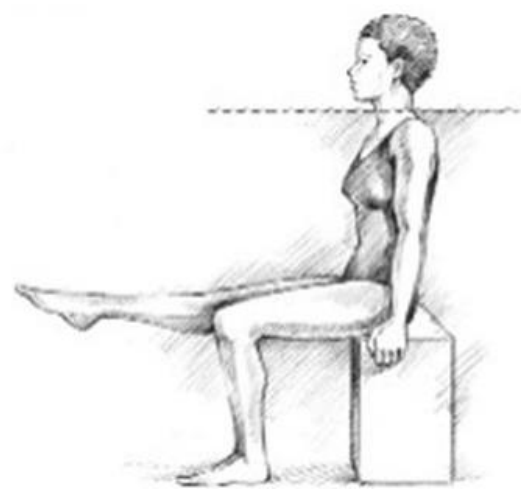

Figura 4. Maniobra de Beatty

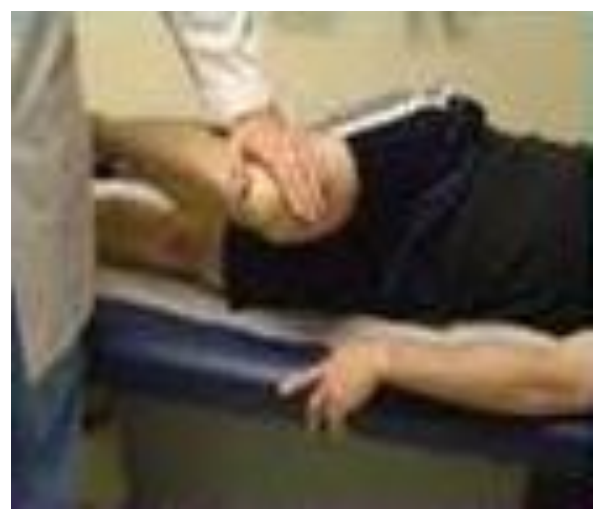

El diagnóstico diferencial debe realizarse con las siguientes patologías:

1) Bursitis isquioglútea: El cuadro doloroso se refiere a la zona glútea y se incrementa con la extensión de la extremidad inferior contra resistencia. Hay dolor a la presión sobre la tuberosidad isquiática. Es frecuente que aparezca en individuos que permanecen sentados largo rato.

2) Bursitis trocantérica: El dolor se refiere a la cara lateral de la cadera que puede irradiar a la extremidad inferior simulando una ciática. El paciente presenta hipersensibilidad a la presión en el trocánter. La abducción activa, la abducción pasiva y la abducción contra resistencia, son dolorosas.

3) Síndrome isquio tibial: Los pacientes refieren dolor en la cara posterior del muslo desde la tuberosidad isquiática hasta el hueco poplíteo, tanto en sedestación como al subir escaleras. Este dolor se incrementa con la flexión de la rodilla contra resistencia, los isquio tibiales están contracturados. 
4) Dolores procedentes de la articulación sacroilíaca: Los pacientes refieren dolor selectivo localizado en zona media glútea. Lasegue negativo. Maniobras de apertura y cierre de la pelvis positivo, con dolor en dicha zona.

5) Radiculopatías: Los pacientes refieren dolor lumbar que irradia por el territorio de la raíz afectada, con disminución de la sensibilidad y de los reflejos según la raíz afectada.

Las posibles causas implicadas en la aparición de este síndrome son: Hiperlordosis lumbar, Hipertrofia del músculo piramidal, fibrosis postraumática, anormalidades anatómicas regionales, artroplastia total de cadera, profesionales que permanecen largos períodos en sedestación, miositis osificante y actividad física intensa, marchadores maratonianos, etc [5-7].

El diagnóstico del síndrome del músculo piramidal es fundamentalmente clínico, ya que no es frecuente obtener hallazgos definitivos en exploraciones complementarias como la Electromiografía (EMG), Tomografía Axial Computarizada (TAC) ni la Resonancia Magnética Nuclear (RMN), estos normalmente sirven para descartar otras patologías.

La ecografía proporciona una correcta visión de las estructuras generadoras de dolor como son músculos, fascias, tendones, periostio y estructuras articulares, lo que ha generado gran interés y motivación entre anestesiólogos y algesiólogos porque ha permitido implementar una herramienta de ayuda para mayor seguridad en los procedimientos de dolor mínimamente invasivos [8, 9].

El bloqueo diagnóstico del músculo piramidal es una de las aplicaciones más útiles de la ecografía [10-12]. Este bloqueo requiere la visualización del músculo piramidal y la punción directa. Esta técnica ha sido descrita con un procedimiento adicional denominado hidrodisección perineural del ciático con solución salina, sin embargo por la necesidad de identificación del nervio ciático por ultrasonido implica aumento en el tiempo del procedimiento y el disconfort del paciente por la punción en la zona pélvica. El objetivo del presente estudio fue describir los resultados de una serie de casos con diagnóstico de síndrome piramidal en los que se aplicó el bloqueo guiado por ultrasonido sin sin hidrodisección perineural del ciático.

\section{Materiales y métodos}

Se describe la técnica y los resultados obtenidos en 10 pacientes del Servicio de Dolor y Paliativos del Instituto Oncológico Nacional "Dr. Juan Tanca Marengo" con diagnóstico clínico de síndrome del músculo piramidal. En los que se infiltró el músculo piramidal teniendo una visión sonográfica adecuada lo que permitió seguridad y fiabilidad del procedimiento. Durante un periodo de 12 meses de febrero 2014 a mayo de 2015, se programaba en un área de bloqueos equipada con un ecógrafo y todas las medidas de reanimación pacientes debían cumplir los criterios habituales de inclusión en un programa 
de procedimientos ambulatorios; no estar en estado de gestación, no presentar alergia a los anestésicos locales y firmar un consentimiento informado; fueron excluidos de este estudio aquellos pacientes con plaquetopenia, signos de infección en área de punción. La valoración del grado de dolor previo y posterior al bloqueo se realizó según la Escala Visual Análoga $(E V A): 0=$ no dolor y $10=$ máximo dolor.

La punción eco-guiada se realizó con normas de esterilidad y de manejo comunes para todos los procedimientos. La sonda ecográfica se limpió antes de cada exploración con un detergente bactericida no abrasivo y se preservó la esterilidad con una funda estéril, el gel conductor también fue estéril y no neurotóxico; la sonda ecográfica se desplazó lentamente para intentar visualizar la imagen más idónea para realizar la punción. Antes de introducir la aguja se realizó una pequeña infiltración del punto de punción con $2 \mathrm{ml}$ de lidocaína al 2 $\%$ utilizando una aguja de $25 \mathrm{G}$. La relación del músculo piriforme con el nervio ciático suele ser variable, pero la mayoría de las veces el nervio transcurre por debajo del músculo en un 78-84 \%, la exploración ecográfica fue muy útil para su visualización y sus relaciones anatómicas; para realizar la infiltración se usaron las referencias anatómicas publicadas previamente [8]. Se evitó la hidrodisección perineural del ciático.

El dolor previo y post-tratamiento se comparó usando estadística no paramétrica para comparar medianas (U de man Whitney).

\section{Resultados}

Esta serie está formada por 10 casos, 9 mujeres y 1 hombre. Con rangos de edad que entre 50 y 70 años de edad. El lado afectado con mayor frecuencia fue el derecho en 5 casos, izquierdo en 3 casos y 2 casos fueron bilaterales, requiriéndose en estos casos infiltración en dos tiempos. Todos los pacientes tuvieron el diagnóstico clínico de síndrome piriforme cuya exploración revelaba maniobra de Pace positiva; irradiación a tercio medio de fémur y rodilla. En 8 casos la Resonancia magnética fue normal y en 2 casos se describió hipertrofia muscular. Las características delos pacientes están descritas en la tabla 1.

El EVA inicial fue $>6$ en 8 pacientes y 2 casos con EVA de 5 (Figura 5). Los pacientes tuvieron una limitación funcional de un 10-20\%. El procedimiento de infiltración fue realizado en los pacientes con éxito sin complicaciones. En ninguno de ellos se realizó hidrodisección perineural del ciático. El tiempo invertido en cada procedimiento fue menor a 22 minutos. Todos los pacientes fueron sometidos a infiltración con corticoides y tratamiento con fisiatría.

El EVA postbloqueo disminuyó hasta 0-1 en todos los casos (Tabla 2). 
Tabla 1. Presentación esquemática de las características clínicas y evolución de los casos

\begin{tabular}{|l|c|c|c|c|c|c|c|c|c|c|}
\hline Caso & $\mathbf{1}$ & $\mathbf{2}$ & $\mathbf{3}$ & $\mathbf{4}$ & $\mathbf{5}$ & $\mathbf{6}$ & $\mathbf{7}$ & $\mathbf{8}$ & $\mathbf{9}$ & 10 \\
\hline Sexo & $\mathrm{F}$ & $\mathrm{F}$ & $\mathrm{F}$ & $\mathrm{F}$ & $\mathrm{M}$ & $\mathrm{F}$ & $\mathrm{F}$ & $\mathrm{F}$ & $\mathrm{F}$ & $\mathrm{F}$ \\
\hline Edad & 57 & 65 & 38 & 68 & 78 & 77 & 59 & 72 & 66 & 70 \\
\hline Lado ran & $\mathrm{D}$ & $\mathrm{D}$ & $\mathrm{D}$ & $\mathrm{I}$ & $\mathrm{D}$ & $\mathrm{I}$ & $\mathrm{D}$ & $\mathrm{B}$ & $\mathrm{I}$ & $\mathrm{B}$ \\
\hline $\begin{array}{l}\text { Dolor en } \\
\text { glúteo } \\
\text { trocánter }\end{array}$ & $\mathrm{SP}$ & $\mathrm{SP}$ & $\mathrm{SP}$ & $\mathrm{SP}$ & $\mathrm{SP}$ & $\mathrm{SP}$ & $\mathrm{SP}$ & $\mathrm{SP}$ & $\mathrm{SP}$ & $\mathrm{SP}$ \\
\hline Pace & - & + & + & - & + & + & - & - & - & + \\
\hline Lasegue & - & - & + & - & - & - & + & - & - & - \\
\hline $\begin{array}{l}\text { Dolor a la } \\
\text { presión }\end{array}$ & + & - & + & + & + & + & + & + & + & + \\
\hline Irradiación & $\mathrm{Rd}$ & $\mathrm{TMF}$ & - & $\mathrm{TMF}$ & - & - & - & - & $\mathrm{TMF}$ & $\mathrm{Rd}$ \\
\hline \begin{tabular}{l} 
Dx. Onco \\
\hline
\end{tabular} & $\mathrm{M}$ & $\mathrm{M}$ & $\mathrm{M}$ & $\mathrm{T}$ & $\mathrm{P}$ & $\mathrm{M}$ & $\mathrm{C}$ & $\mathrm{M}$ & $\mathrm{T}$ & $\mathrm{M}$ \\
\hline
\end{tabular}

D: derecho, I: izquierdo, B: bilateral. TMF: tercio medio de fémur. Dx: diagnóstico. TX: tratamiento, Onco: oncológico. EVA: escala visual análoga, RMN: resonancia magnética nuclear, TAC: tomografía computada, RD: Rodilla. P: Cáncer de Próstata. M: Cáncer de mama. T: cáncer de tiroides. C: cáncer de cérvix.

Tabla 2. Mediana de Dolor de los pacientes pre y post tratamiento

\begin{tabular}{|l|c|c|c|}
\hline & $\begin{array}{c}\text { EVA pre- } \\
\text { tratamiento } \\
\mathrm{n}=10\end{array}$ & $\begin{array}{c}\text { EVA post- } \\
\text { tratamiento } \\
\mathrm{n}=10\end{array}$ & $\begin{array}{c}\text { P (U de man } \\
\text { Withney) }\end{array}$ \\
\cline { 1 - 3 } P50 & 6 & 0 & \multirow{2}{*}{$<0.001 *$} \\
\hline P25 & 5.75 & 0 & \\
\hline P75 & 6.25 & 1 & 0.25 \\
\hline
\end{tabular}

P50: mediana. P25: percentil 25, P75 percentil 75.

En esta serie ningún paciente de los incluidos tenía metástasis óseas descartadas con gammagrafía ósea, siendo el mayor porcentaje pacientes sin actividad tumoral, pero con evidente proceso de desmineralización ósea causante de su algia. No se realizaron electromiografías en los pacientes. Los estudios de Resonancia Magnética descartaron patologías artrósicas y/o degenerativas. En este reporte el cáncer de mama fue el diagnóstico oncológico más prevalente en 6 de 10 casos. 
Figura 5. Gráfico de barras de error de la media de EVA pre y post tratamiento en pacientes con Síndrome Piramidal

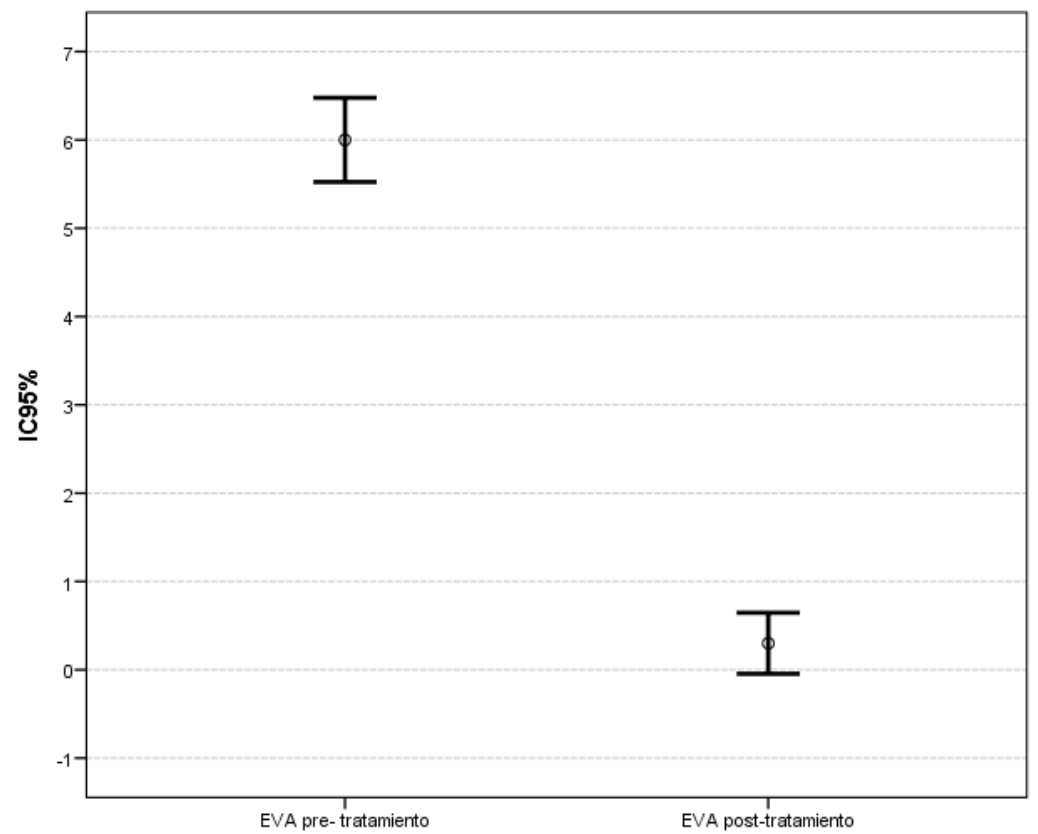

\section{Terapia física}

Todos los pacientes recibieron 3 sesiones semanales de ejercicios de estiramiento del músculo piriforme, isquiotibial y de los de los músculos extensores de la cadera para disminuir los síntomas de dolor, limitación y recuperar la amplitud de movimiento de la articulación afecta. La intensidad del estos ejercicios fue disminuyendo a medida que mejoraron los síntomas.

En la segunda fase no dolorosa se aplicó técnicas manuales para liberar las articulaciones lumbo-pélvicas y del miembro inferior y devolver la completa movilidad al resto de estructuras musculo-ligamentosas. Dentro de las técnicas de terapia física, los estiramientos del músculo, junto con los masajes locales, los ultrasonidos y la estimulación eléctrica transcutánea fueron aplicados a todos los pacientes para obtener una mejoría del cuadro doloroso adicionalmente a la infiltración guiada por ecografía.

\section{Discusión}

En esta serie de casos se presenta el manejo del dolor dado a pacientes que cumplían el criterio de síndrome piriforme en quienes se utilizó la infiltración de esteroides guiada por ecografía. Al respecto en la literatura médica se describe que los tratamientos conservadores son suficientes en la mayoría de los casos para el control del dolor [9-11], sin embargo cuando estos tratamientos no son efectivos deben emplearse técnicas más dirigidas como los bloqueos con esteroides, anestésicos locales e incluso con toxina botulínica, con lo que el alivio del dolor disminuye hasta en un $95 \%[12,13]$. Se utilizó la 
técnica de inyección directa al músculo piriforme evitando la hidrodisección perineural del ciático. El tiempo invertido en cada procedimiento fue menor a 22 minutos y ningún procedimiento desarrolló complicaciones haciendo muy sencilla la técnica y segura.

En la presente serie no se utilizó toxina botulínica todos los casos fueron tratados con esteroides. En la presente serie de casos no se realizaron cirugías para sección del músculo piramidal, aunque se ha descrito que la cirugía debe considerarse cuando fracasan los medios anteriores y consistirá en general en realizar una sección del músculo piramidal, con una neurolisis del nervio ciático [9-10].

El dolor a la presión en la zona del músculo piramidal fue el signo más prevalente en todos los casos en esta serie, luego fue el signo de Pace. Al respecto el diagnóstico de síndrome piriforme es una causa común de dolor lumbar, glúteo y del muslo, frecuentemente asociado con síntomas del nervio ciático. Las posibles etiologías incluyen lesión muscular o estiramiento muscular crónico asociado con trastornos de la marcha. Existe una vía final patológica común que incluye hipertrofia, espasmo, contractura, inflamación y cicatrización del músculo piriforme, lo que lleva a la compresión del nervio ciático. Todavía está pendiente relacionar una vía inflamatoria paraneoplásica en pacientes con neoplasias y verificar si es más prevalente en la población oncológica.

Este estudio tiene debilidades al ser una serie de casos, debido a la incidencia baja del síndrome piramidal en un grupo de pacientes en un centro de manejo de dolor oncológico. Adicionalmente no se aplican técnicas de disección ecográfica previo a la inyección del esteroide. Próximos estudios deberán incluir la posibilidad de tratamiento con toxina botulínica comparadas caso control con infiltraciones de esteroides para los pacientes con síndrome piramidal en pacientes con y sin hidrodisección perineural del ciático.

\section{Conclusiones}

En la presente serie de casos el síndrome piramidal fue controlado satisfactoriamente con infiltración de esteroides sin hidrodisección perineural del ciático y terapia física.

\section{Agradecimientos}

Queremos agradecer el apoyo institucional para la realización del estudio así como los trabajadores y pacientes del Instituto Oncológico Nacional "Dr Juan Tanca Marengo"-Solca Guayaquil. 


\section{Información adicional}

Abreviaturas

Solca: Sociedad de Lucha Contra el Cáncer.

EMG: Electromiografía.

TAC: Tomografía Axial Computarizada.

RMN: Resonancia Magnética Nuclear

\begin{abstract}
Nota del Editor
La Revista Oncología Ecu permanece neutral con respecto a los reclamos jurisdiccionales en mapas publicados y afiliaciones
\end{abstract} institucionales.
Archivos Adicionales

Ninguno declarado por los autores.

Fondos

Los fondos para la presente investigación fueron propios de los autores del presente artículo.

\section{Disponibilidad de datos y materiales}

Existe la disponibilidad de datos bajo solicitud al autor de correspondencia. No se reportan otros materiales.

\section{Contribuciones de los autores}

MVM, NL idea de investigación, MPy JG realizaron la revisión bibliográfica. MVM, MP Y JG compilaron los datos. MP y JG escribieron el artículo. MVM y NL realizaron el análisis crítico del estudio. Todos los autores aprobaron la versión final del manuscrito.

Aprobación de ética y consentimiento para participar

No se aplica a un estudio observacional.

\section{Consentimiento para publicación}

No aplica a un estudio observacional. 
Mariana Vallejo Martínez, Médica tratante del Servicio de Dolor y Paliativos del Instituto Oncológico Nacional "Dr. Juan Tanca Marengo".-Solca- Guayaquil. Master en Atención paliativa.

https://orcid.org/0000-0001-5727-262X

Nancy Lino, Jefe Servicio de Cuidados Paliativos y Manejo del Dolor del Instituto Oncológico Nacional "Dr. Juan Tanca Marengo".Solca- Guayaquil.

\section{Palma, Postgradista de Anestesióloga R4,}

J. Guamán, Lcdo en fisitría del Instituto Oncológico Nacional "Dr. Juan Tanca Marengo".Solca- Guayaquil.

Revisiones por pares

Acceda a la revisión de pares académicos en el siguiente enlace: https://publons.com/review/3147327

\section{Referencias}

Abreviaturas en la referencias

DOI: Digital Object

Identifier

PMID: PubMed Identifier

SU: Short URL
1. Domingo T, Mayoral V, Casals M, Serrano A, Miguel M, Sabaté A. La punción guiada con ultrasonidos aplicada a una unidad de dolor crónico. Rev.Esp. Anestesiologia. Reanimacion; 2010;57(8):470-543.DOI: $\underline{10.1016 / \text { S0034-9356(10)70710-6 }}$

2. Fanucci E, Masala S, Squillaci E, Sodani G, Varrucciu V, Ursone A, Simonetti G. Pyriformis muscle syndrome: CT/MR findings in the percutaneous therapy with botulinic toxin. Radiol Med. 2003;105(12):69-75. PMID:12700548.

3. Filler AG, Haynes J, Jordan SE, Prager J, Villablanca JP, Farahani K, et al. Sciatica of nondisc origin and piriformis syndrome: diagnosis by magnetic resonance neurography and interventional magnetic resonance imaging with outcome study of resulting treatment. J Neurosurg Spine. 2005 Feb;2(2):99115. DOI: $10.3171 /$ spi.2005.2.2.0099

4. Lang AM. Botulinum toxin type B in piriformis syndrome. Am J Phys Med Rehabil. 2004;83(3):198-202. DOI: $10.1097 / 01 . P H M .0000113404 .35647 . D 8$

5. Cass SP. Piriformis syndrome: a cause of nondiscogenic sciatica. Curr Sports Med Rep. 2015 Jan;14(1):41-4. DOI: 10.1249/JSR.0000000000000110.

6. Tonley JC, Yun SM, Kochevar RJ, Dye JA, Farrokhi S, Powers CM. Treatment of an Individual with piriformis syndrome focusing on hip muscle strengthening and movement reeducation: a case report. J Orthop Sports Phys Ther. 2010;40(2):103-111. DOI: 10.2519/jospt.2010.3108

7. Ruiz-Arranza JL, Alfonso-Venzaláb, Villalón-Ogayarc J. Síndrome del músculo piramidal. Diagnóstico y tratamiento. Presentación de 14 casos. Rev. esp. cir. ortop. traumatol. 2008;52:359-65. SU: goo.gl/mQiEeo

8. Bevilacqua A, Diz Villar A, Curt F, Illodo G, Refojos FJ, López JM. Ultrasound-guided piriformis muscle injection. A new approach. Rev Esp Anestesiol Reanim. 2016;63(10):594-598. DOI: 10.1016/j.redar.2016.02.008.

9. Park $\mathrm{CH}$, Lee SH, Lee SC, Park HS. Piriformis muscle: clinical anatomy with computed tomography in korean population. Korean J Pain. 2011;24(2):87-92. DOI: 10.3344/kjp.2011.24.2.87 
10. Lijec V. Piriformis muscle syndrome: etiology, pathogenesis, clinical manifestations, diagnosis, differential diagnosis and therapy. 2013;135(1-2):33-40. PMID: 23607175

11. Rossi $P$, Cardinali $P$, Serrao M. Parisi L, Bianco F, De Bac S. Magnetic Resonance Imaging Findings in Piriformis Syndrome: A Case Report. Arch Phys Med Rehabil 2001;82(4):519-21. DOl: 10.1053/apmr.2001.21971

12. Lang AM. Botulinum toxin type B in piriformis syndrome. Am J Phys Med Rehabil. 2004;83(3):198-202. PMID: 15043354

13. Michel F, Decavel P, Toussirot E, Tatu L, Aleton E, Monnier G, et al. Piriformis muscle syndrome: diagnostic criteria and treatment of a monocentric series of 250 patients. Ann Phys Rehabil Med. 2013;56(5):371-83. DOI: 10.1016/j.rehab.2013.04.003 